\title{
ThoughtMix: Interactive Watercolor Generation and Mixing Based on EEG Data
}

\author{
R.P.C. Janaka Rajapakse \\ Graduate Institute of Animation and Film Art, Tainan National University of the Arts, No. 66, Daci, \\ Guantian District, Tainan 72045, Taiwan
}

\author{
Yoshimasa Tokuyama \\ Department of Media and Image Technology, Tokyo Polytechnic University, 1583, Iiyama, Kanagawa 243-0297, Japan \\ E-mail:janakaraja@gmail.com,tokuyama@mega.t-kougei.ac.jp, \\ ${ }^{*}$ www.tnnua.edu.tw and ${ }^{\dagger}$ www.t-kougei.a.cjp
}

\begin{abstract}
In modern art, new types of installations and artworks use sensor-based inputs as an interactive method to create new forms of media art. Most of those installation artworks have static and pre-captured contents; they tend to be played as dynamic, and by some concerned as interactive creations. However, most installations are not able to capture the visitor's rich feedback which could trigger the contents. In order to identify the relation between the cyberneticaesthetics and visitor's brain wave activities, we propose an electroencephalogram (EEG) data-driven approach to generate watercolor and mixing effects which visualizes the changes in brain wave activities in real-time. Our proposed application transforms the brain waves into real-time watercolor effects that are displayed by generating the data about emotion, attention, meditation, and neural mechanisms with EEG data. "ThoughtMix" creates watercolor effects as an immersive experience to influence creativity and art therapy by actually visualizing the colors the user's mind creates.
\end{abstract}

Keywords: EEG art, watercolor, thought colors, digital colors, interactive mix, interactive generation

\section{Introduction}

Currently, people living in a digitally connected era are in the space of reality and virtuality. Digital technology is commonly used for human-computer interaction and content creations [1]. Interactive art is a method that stimulates a variety of the user's senses and activities inside digitally created contents with a computer, so that users may interactively experience environments and spaces that can be difficult to create in traditional techniques [2]. As computer graphics-based applied art and technology becomes advanced and as various sensor hardware interfaces are developed and spread, a variety of applications that enable the user to interactively encounter spatial and temporal experiences are being created [3], [4]. Additionally, there is a growing demand for art and technology that support these interactive applications [5].

It is important that digital art technology moves beyond the boundaries of the traditional interaction techniques to provide a creative space that allows the user to create and experience therapeutic sensations. The acceptance of brain-computer interface (BCI) leads many art researchers to explore the diversity of mind cybernetic methods and expression forms in electroencephalogram (EEG) art [6]. This paper presents an EEG data-driven approach to generate real-time watercolor and mixing effects that visualize the changes in brain wave activities in order to identify the relation between the cyberneticaesthetics and user's brain wave activities. The proposed application "ThoughtMix" transforms the brain waves into real-time watercolor effects that are displayed by generating the data about emotion, attention, meditation,

(C) The 2021 International Conference on Artificial Life and Robotics (ICAROB2021), January 21 to 24, 2021 
and neural mechanisms with EEG data. This interactive application creates watercolor effects as an immersive experience to influence creativity and art therapy by actually visualizing the colors the user's mind creates.

The paper is organized as follows: section 2 presents related work. Section 3 describes our development framework. Section 4 is devoted to experimental results. Section 5 discusses the main points presented throughout the article, drawing conclusions on future research directions.

\section{Background}

There are several works in literature that cover interactive art in various forms. Most papers feature contents and comparison methods between different generative techniques, providing performance results of the interactive techniques, and often reports of users' preferences. Unfortunately, due to the variety of different art installations and different interfaces used for interaction and control, it is still difficult to derive visitorgenerated contents. Therefore, further research is necessary to understand which are the best options for a specific immersive experience to influence creativity and therapeutic sensations.

\subsection{EEG in Art}

When compared to traditional interactive feedback techniques, using EEG as a biological control is considered relatively implicit. Mechanical operation and motion detection are widely used in traditional interactive art applications. In these traditional applications, the explicit macro-communication generated is based on the participant's movements and behaviors. However, EEG control directly uses electrodes to acquire the activity of neurons and takes thinking as a controller. EEG artwork is a new form of communication that cannot be observed through body behavior but the mind.

Prpa and Pasquier [6] defined a structured overview of the expanding field of BCI art, with utilized approaches and BCI devices, and proposed a systematic way of categorizing artworks based on their similarities in the taxonomy. This presented taxonomy encompasses sixty-one artworks that illustrate the nuances of the categories in the taxonomy. According to the distance between input and output control, Fishkin's [7] introduced a classification of the perceptive interface based on the level of self-containment. Wadeson et al. [8] provided different views on creative control, which are separated into four categories for participants: passive, selective, direct, and collaborative. These classification models described the spatial relationship between the existences of participants and artwork, which determine the behavioral characteristics of aesthetic construction. In this paper, we focus on passive inputs which does not require the participant to perform any particular task to change or influence their brain activity explicitly.

\subsection{Generative Art: Color and Mixing}

Computer-based generative art is a procedure to create artforms by executing a set of rules in a methodical collaboration between an artist and a machine [10]. Galanter [9] defined "Generative art refers to any art practice where the artist uses a system, such as a set of natural language rules, a computer program, a machine, or other procedural invention, which is set into motion with some degree of autonomy contributing to or resulting in a completed work of art". Shuhei Matsuyama [11] created an installation "dyebirth" that continually creates organic patterns through digitally controlled physical phenomena produced by a mixture of water, ink, and chemical substances.

Refik Anadol [12] presented an installation artwork named "Melting Memories" based on measured EEG data, which offered new insights into the representational possibilities emerging from the intersection of advanced technology and contemporary art. "Thinking in Color" was an interactive experience that used an EEG brainwave scanner to generate colors, influenced in realtime by the user's brainwave activity [13]. To generate watercolor effects, the proposed application in this paper, named "ThoughtMix" also focused on real-time EEG data and generative art techniques.

\section{Development Framework}

In the proposed development framework, the EEG-based interaction approach handles brainwave inputs without additional equipment. In order to acquire EEG data, MindWave Mobile 2 EEG headset [14] device was chosen because of its economic affordability, reliability in the delivery of data related to the user's mental states, ease of use, and the comfort of the user feel when interacting with the device. Figure 1 shows how the EEG 
headset integrates with the TouchDesigner [15] to create a development environment. MindWave Mobile 2 EEG headset was connected to the computer via Bluetooth. Real-time EEG data acquisition, signal processing, and classification were used in ThinkGear connector installed on the computer that sent the classification data through a user datagram protocol (UDP) over the local network to the computer running TouchDesigner software which generates contents.

\subsection{Contents creation}

To generate watercolor effects, TouchDesigner was used as a visual programming environment. The Noise texture operation (TOP) generates a variety of noise patterns

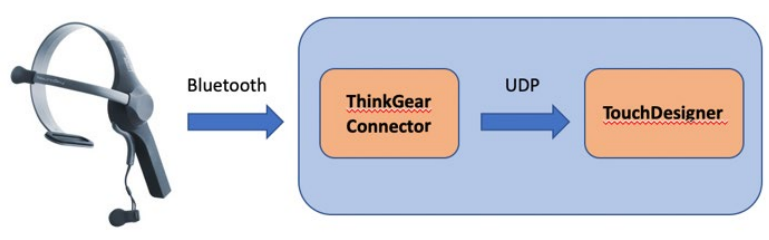

Fig. 1. Block Diagram of Brainwave Controlled

including Perlin, simplex, sparse, alligator, Hermite, and random which often used to procedurally generate organic surface textures and real-time effects. The Noise texture operation and the displacement texture operation nodes were used to create watercolor effects (Figure 2). In TouchDesigner texture operations, Slope-TOP used horizontal luminance and vertical luminance for Red and Blue channels while Green channel was kept neutral. Blur-TOP used as a filter with 5-pixel size, and Level$T O P$ used to quantization brightness and contrast.

\subsection{Mapping colors}

As literature described [6], the direct mapping technique was used. The classified eight EEG band power values are mapped to the colors as shown in Table 1. High Alpha and High Beta mapped into translate sampling parameters of Y-axis and Z-axis in Noise TOP (Simplex 3D GPU) which produce real-time dynamic noise patterns.

Table 1. EEG bands, their frequencies and colors
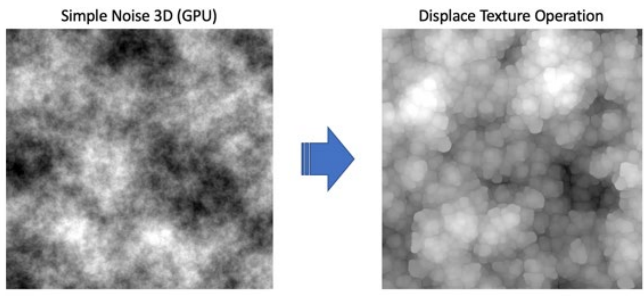

Fig. 2. The Noise-TOP and the Displace-TOP can be generated in TouchDesigner.

\begin{tabular}{ccc} 
Theta & $4-7 \mathrm{~Hz}$ & Orange \\
Low Alpha & $8-9 \mathrm{~Hz}$ & Yellow \\
High Alpha & $10-12 \mathrm{~Hz}$ & Green \\
Low Beta & $13-17 \mathrm{~Hz}$ & Light Blue \\
High Beta & $18-30 \mathrm{~Hz}$ & Blue \\
Low Gamma & $31-40 \mathrm{~Hz}$ & Indigo \\
High Gamma & $41-50 \mathrm{~Hz}$ & Violet \\
\hline
\end{tabular}

According to the ThinkGear Serial Stream SDK, the acquired eSenses Attention meter value is reported on a relative eSense scale of 1 to 100 which can be categorized into five different levels. However, this application used an indirect mapping technique which categorized a value between 0 to 40 at any given moment in time is considered "reduced". A value between 41 to 100 indicates "increased" levels of the eSense. The two states represent 0 and 1 , which operates the reset parameter of the Feedback texture operation (Feedback TOP [15]) node. The Feedback TOP can be used to create a feedback effect that gives motion blur without cleaning the color buffer. When the "reduced" state, Feedback TOP passes through the image connected to input colors generated by EEG band values.

\section{Experimental Results}

As a pilot experiment, the ThoughtMix application was tested with graduate students from Tainan National University of the Arts. Figure 3 shows some examples of the participant's experiences and the effects created by their own brain waves, the left column shows dynamic attention and the right column shows stable and enhanced attention.

\footnotetext{
\begin{tabular}{ccc}
\hline EEG band & Frequency range & Mapped Colors \\
\hline Delta & $1-3 \mathrm{~Hz}$ & Red
\end{tabular}

(C) The 2021 International Conference on Artificial Life and Robotics (ICAROB2021), January 21 to 24, 2021
} 


\section{Conclusion}

The main objective of this application is to provide an immersive experience to influence creativity and art therapy by visualizing the watercolor effects the user's mind creates. Despite the limited user study, the system successfully generated and incorporated EEG data which allows the user to identify the relation between the cybernetic-aesthetics and brain wave activities.
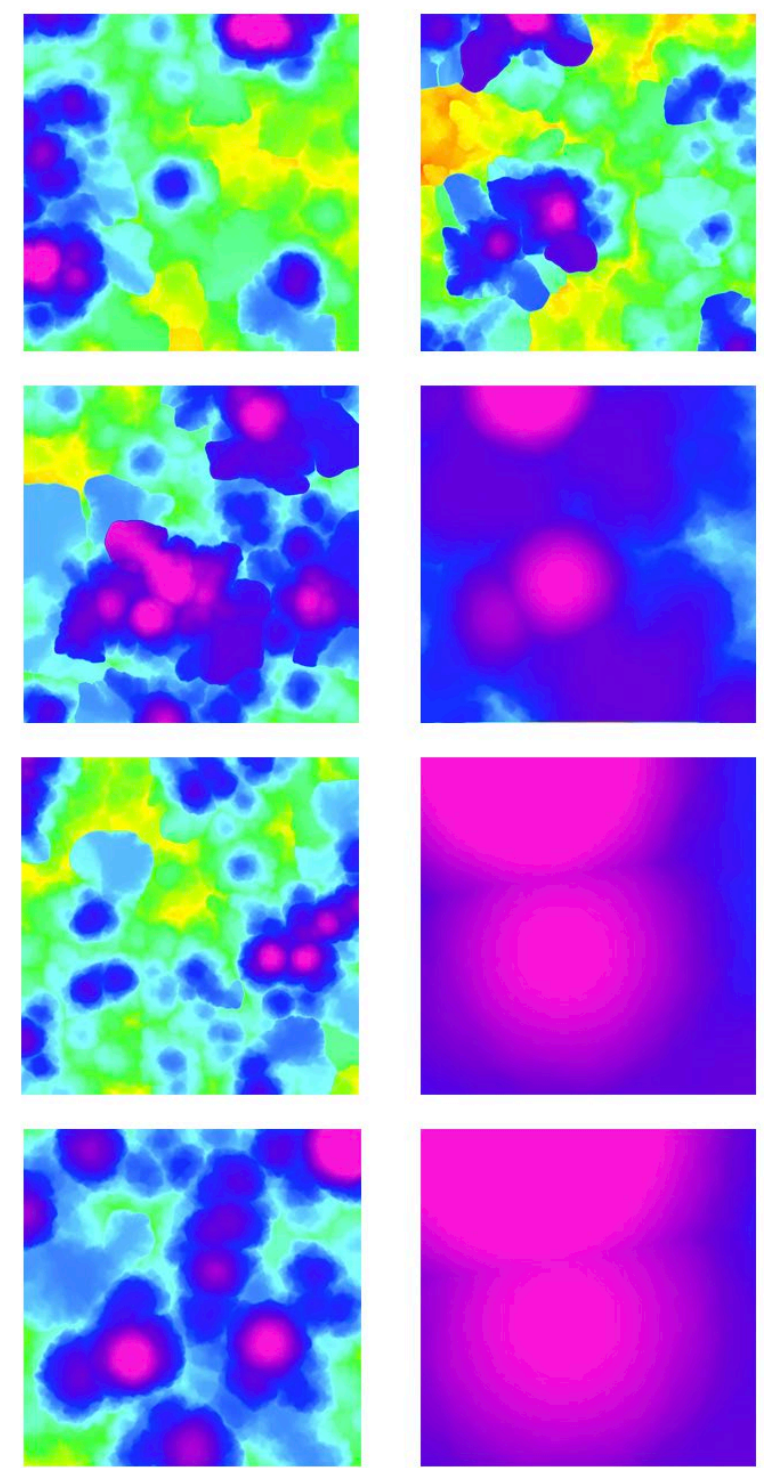

Fig. 3. Some examples of the participant's experiences and the watercolor effects created by their own brain waves.

\section{References}

1. S.U. Ahmed, A. Al Mahmud, K. Bergaust (2009) Aesthetics in Human-Computer Interaction: Views and Reviews. In: Jacko J.A. (eds) Human-Computer Interaction. New Trends. HCI 2009. Lecture Notes in Computer Science, vol 5610. Springer, Berlin, Heidelberg. https://doi.org/10.1007/978-3-642-02574-7_63

2. E. Edmonds, G. Turner, L. Candy, Approaches to interactive art systems. In: Proceedings of the 2nd International Conference on Computer Graphics and Interactive Techniques in Australasia and South East Asia, pp. 113-117, 2004.

3. Z. Wang, Theory of Digital Interactive Art Creation. Shanghai University, Shanghai (2011)

4. J. Strehovec, New media art as research: art-making beyond the autonomy of art and aesthetics. Technoetic Arts, Vol. 6, No. 3, pp. 233-250, (2009). https://doi.org/10.1386/tear.6.3.233_1

5. Suzanne Dikker, Matthias Oostrik, Peter Burr, Diederik Schoorl, and Matthew Patterson Curry. 2014. Mutual Wave Machine. Art. Retrieved May 3, 2019 from https://mai.art/content/mutual-wave-machine

6. M. Prpa, P. Pasquier, Brain-Computer Interfaces in Contemporary Art: A State of the Art and Taxonomy, Brain Art. Springer, (2019), https://doi.org/10.1007/9783-030-14323-7 3

7. K. P. Fishkin, A taxonomy for and analysis of tangible interfaces. Personal and Ubiquitous Computing, Vol. 8, No. 5, pp. 347-358 (2004)

8. A. Wadeson, A. Nijholt, C. S. Nam, Artistic braincomputer interfaces: state-of-the-art control mechanisms. Brain-Comput. Interfaces Vol. 2(2-3), pp. 70-75, (2015).

9. P. Galanter, "What is generative art? Complexity theory as a context for art theory." In GA2003-6th Generative Art Conference. 2003

10. A. Dorin, J. McCabe, J. McCormack, G. Monro \& M. Whitelaw (2012) A framework for understanding generative art, Digital Creativity, Vol. 23, No. 3-4, pp. 239-259, DOI: 10.1080/14626268.2012.709940

11. Shuhei Matsuyama, dyebirth: An installation work, https://nor.tokyo/dyebirth, Accessed on 10th Dec 2020.

12. Refik Anadol. 2018. Melting Memories. Installation Art, http://refikanadol.com/works/melting-memories/ Accessed on 10th Dec 2020.

13. Thinking in Color, https://www.lbbonline.com/news/thisexperience-uses-brain-waves-to-create-colour, Accessed on 10th Dec 2020.

14. NeuroSky, ThinkGear Connector, http://developer.neurosky.com/docs/doku.php?id=thinkge ar connector tgc. Accessed on 10th Dec 2020.

15. TouchDesigner, https://derivative.ca/, Accessed on 10th Dec 2020. 